\title{
ANÁLISIS TOPONÍMICO DE KENT: UNA CONTRIBUCIÓN FONOLÓGICA A LA DIALECTOLOGÍA BAJOMEDIEVAL INGLESA ${ }^{1}$
}

\author{
$M^{a}$ Auxiliadora Martín Díaz. Universidad de la Laguna
}

\begin{abstract}
El interés del Kéntico (o Kentio) reside en que, a lo largo de su historia, ha mostrado siempre características peculiares que lo han singularizado como una variedad distintiva dentro del inglés sureño y que posiblemente se atribuyen al hecho de que Kent está en una zona de tránsito que limita al noroeste con la emergente ciudad de Londres, y al haber sido escenario de una amalgama de culturas (sajones, frisios, francos y jutos). Nuestra atención se centra aquí en ilustrar la aportación que desde la toponimia, concretamente desde el análisis formal de ciertas variables del Inglés Antiguo relevantes en el dialecto Kéntico, se puede hacer a la dialectología bajomedieval.
\end{abstract}

\section{EL DIALECTO DE KENT}

El Kéntico se conoce ya desde el s. VIII por los nombres propios que aparecen en algunos breves documentos latinos. En el s. IX se utiliza en valiosos documentos de origen vernáculo (Kentish Charters). Además de éstos, ciertas glosas que datan del año 900 hechas a la Historia Ecclesiastica de Beda parecen contener elementos del Kéntico, así como otros cuatro documentos latinos que también parecen recoger algunos nombres. Ya en el período tardío del Inglés Antiguo (IA), este dialecto es conocido por los Kentish Psalms, el Kentish Hymn, y las glosas hechas a los Proverbs $(K G)$, todos ellos del s. X tardío. Para el período bajomedieval, la publicación del Linguistic Atlas of Late Medieval English (LALME) ha conseguido enriquecer nuestro conocimiento de las fuentes kénticas que van desde textos literarios cuyo origen es perfectamente conocido (anchor texts), como el Ayenbite of Inwyt de Dan Michel of Northgate (1340), los Sermones de John Sheppey, las Religious Lyrics del s. XIV, o los poemas de William of Shoreham a textos literarios o

\footnotetext{
1 Este artículo es parte de un proyecto de investigación titulado "Diccionario nuclear sintáctico de base semántica del léxico del inglés antiguo", financiado por el Gobierno Autónomo de Canarias (No. PI 1999/ 136).
} 
documentales que pertenecen a la categoría de localizable texts entre los que destacan las Chartae Antiquae de los Archivos y Biblioteca de la Catedral de Canterbury, el MS. Takamiya 3, los Kentish Sermons de la Bodleian Library, y el 'Merlin', de la Cambridge University Library².

En cuanto a la consideración social de nuestro dialecto, hemos de señalar que durante el s. XIV los movimientos migratorios, procedentes sobre todo de East Anglia y las East Midlands, provocaron que el dialecto de Londres, de fuerte base sureña, se viera marcadamente influido por el dialecto de las Midlands orientales (Morsbach, 1888: 161; Luick, 1914/1941: §36; Wyld, 1927: 50-53; Samuels, 1972: 166). Desde un punto de vista lingüístico, la sociedad londinense comienza pues a fluctuar entre esta variedad dialectal y el dialecto propio del sureste, siendo este último el que preferentemente se utilizó entre los hablantes londinenses con una posición social baja. Ello explica el desdén con que se la considerará, desde otras esferas, a partir de este momento (Blake, 1981: 47). La actitud despectiva de la alta sociedad londinense hacia una variedad que consideran vulgar se extenderá por el sur hasta llegar a ser asumida por los hablantes del marginado dialecto de Kent. Nacer en Kent y hablar el Kéntico en esta época es indicativo, por tanto, de un origen provinciano carente de todo prestigio social.

Desde un punto de vista fonológico, la tradición señala como rasgos típicos del sureste (y por tanto no sólo de Kent) la apertura y pérdida de labialización del sonido del IA y, la tendencia de la $c e$ del IA a cerrarse, la sonorización de las fricativas sordas iniciales y la representación gráfica de los diptongos del IA eo y ea como dígrafos. Wyld (1927) distingue además una evolución singular de la $e$ del IA hacia $e$ en Kent, así como una evolución hacia -en cuando la $c_{2}$ precede a una nasal. Según Brook (1965) son también rasgos típicos de Kent, la pérdida de la aspiración en la combinación $h l$ - y la representación de la $o$ del IA como uo. Para Mossé (1952) la vocalización de $g$ ante las vocales $a, o, u$ y las líquidas $l, r$ no sucede hasta el 1400 en Kent. Dentro de los estudios de corte más estructuralista, Hogg (1992) habla de un desarrollo peculiar de las vocales frontales kénticas como contrapunto a lo sucedido en el sajón occidental.

\section{LA DIALECTOLOGÍA MEDIEVAL INGLESA: EL ENFOQUE TOPONÍMICO}

Importantes en la investigación dialectal de la lengua inglesa medieval fueron las tempranas pero valiosas contribuciones de Oakden (1930), y Moore, Meech y Whitehall (1935), que aunque de forma exploratoria (dado el bajo número de criterios usados y de fuentes consultadas) permitieron establecer una radiografía dialectal del país en su totalidad. Sobresalen también los tradicionales estudios monográficos descriptivos, dedicados en su mayoría al análisis de ciertas obras relevantes, y que normalmente reflejaban la distribución regional de algunas variantes textuales.

Siguiendo el enfoque geográfico, tenemos además el Linguistic Atlas of Late Mediaeval English (LALME: 1986), obra de referencia actual y completa dentro de la investigación dialectal y que, asimismo, adopta una metodología con la que poder lograr una imagen más exacta y rica de la variación medieval inglesa ${ }^{3}$.

\footnotetext{
${ }^{2}$ La enumeración de otros setenta documentos, dentro del apartado de local documents, y, por otro lado, los associated literary manuscripts completan la contribución aportada por el Atlas a nuestro conocimiento de las fuentes de interés para el estudio del Kéntico bajomedieval.

${ }^{3}$ Gracias al diseño de un complejo cuestionario de criterios fonológicos y morfológicos McIntosh, Samuels y sus colaboradores pudieron trazar un mapa de las variantes lingüísticas de un número de anchor texts (textos ancla cuya localización es conocida) pertenecientes al período 1350-1450, que a su vez, al ser
}

Odisea, $\mathrm{n}^{\mathrm{0}} 2,2002$ 
Durante el s. XX también se desarrolla el enfoque onomástico, de acuerdo con el cual los topónimos, entre otros nombres, se analizan con el fin de extraer información sobre el comportamiento de ciertas distinciones fonológicas básicas. Dentro de este enfoque destacan, entre otros, Ekwall (1931); Arngart (1949); Rubin (1951), Sundby (1963), y Kristensson (1967), (1987), (1995) y (2001). Este último autor a mediados de siglo se lanzó a realizar un proyecto, aún inacabado, para analizar los dialectos del IM de manera individual en un período que abarca de 1290 a 1350. Entre los proyectos dedicados a la investigación onomástica del condado que nos ocupa, tan sólo contamos con Ek (1972) y (1975) y Kristensson (2001), los cuales se concentran en el análisis específico de las vocales ${ }^{4}$ para el inglés del sureste (Kent incluido) durante el período medio. Sin embargo todavía carecemos de los volúmenes correspondientes a Kent entre los volúmenes de la English Place-Name Society $(E P N S)^{5}$.

En resumen, dentro de los estudios modernos sobre los dialectos medievales podemos distinguir al menos tres tipos de acercamiento que, en lo que se refiere al Kéntico, se ilustran de la manera siguiente:

i. Los estudios monográficos basados en una obra representativa, a ser posible hológrafa de la que se presuponía una evidencia más precisa (i.e., las observaciones y el aparato crítico incluido por Pamela Gradon (1979) en su estudio detallado del Ayenbite of Inwyt de Dan Michel).

ii. Trabajos dedicados al análisis global de un conjunto de variables dialectales en relación con un número amplio y variado de fuentes. En este sentido son dignas de mención las investigaciones realizadas por Heuser (1895), Luick (1914/1941), Jordan-Crook (1925/1974); Wyld (1927); Oakden (1930); Moore, Meech y Whitehall (1935); Mossé (1952) y Brook (1965).

iii. Estudios que analizan alguna variable en concreto, o alguna característica dialectal en particular, bien a partir de textos literarios (Taylor, 1924), bien basándose en estudios onomásticos en general, o toponímicos en particular. Aunque no exclusivamente para Kent, tenemos a Serjeantson (1922), Ek (1972) y (1975) y Kristensson (2001); o estudios donde la información aportada por los textos literarios hológrafos y por las formas toponímicas se combina (Serjeantson, 1927b).

El estudio dialectal de los topónimos que se pretende llevar a cabo aquí, aunque no totalmente ignorado, está lejos de haberse agotado pues aún hay documentos cuya información dialectal no ha sido completamente explotada. En el propio LALME, por ejemplo, de las fuentes utilizadas para el condado de Kent una gran mayoría corresponde, según palabras de Benskin, a textos como "reports of local documents", o "short literary texts", en los que Samuels, "expecting rather little from them, he recorded rather less" (1991: 212-13). Además, es precisamente la existencia de autores como Ekwall, Rubin, Ek o Kristensson, lo que me impulsa a reiterar la importancia del estudio toponímico de un gran número de condados ingleses que aún queda por realizar.

tomados como puntos de referencia, permitieron la localización, mediante la fit-technique (McIntosh et al., 1987; Benskin, 1991) de otros textos de la época.

${ }^{4}$ Concretamente Ek se centra en la $y$, la $a$ y el diptongo eo y Kristensson en las vocales en general pero excluyendo los diptongos.

5 Véase al respecto Martín Díaz (2001).

Odisea, $\mathrm{n}^{\circ} 2,2002$ 


\section{OBJETIVOS}

El objetivo fundamental de este trabajo es el querer contribuir, a través del estudio toponímico del Kéntico bajomedieval, a un mejor conocimiento de la configuración dialectal del Inglés Medio (IM) en general. El alcance del mismo es en sí limitado pues supone el estudio de una zona geográfica relativamente pequeña, en el que se presta atención únicamente al análisis de algunas variables clave dentro de unos topónimos que, con frecuencia, se consideran arcaicos y algo oscuros en su origen. Sin embargo, dichas consideraciones no deben menoscabar su interés porque:

i. En la práctica se admite el análisis dialectológico de un área cuyas fronteras físicas vienen determinadas por la intersección o acumulación de una serie de isoglosas (Milroy, 1992: 186-7).

ii. "The material which has so far proved most profitable for the investigation of OE and ME dialects consists of place-names" (Kristensson 1967: XI), y es gracias a investigaciones de esta naturaleza como podemos constatar que "There are sounddevelopments which are not attested at all in literary texts but are inferred solely on the evidence of name-forms" (Kristensson, 1976: 58).

iii. Los topónimos tienen a su favor la precisión en la localización y el hecho de que, en lo que a Kent se refiere, el análisis dialectológico de su toponimia no ha sido aún satisfactoriamente resuelto.

Los topónimos tienden a ser conservadores y a preservar formas que incluso puedan haberse perdido ya en la lengua hablada ordinaria. Esto explica que el análisis de la distribución de las realizaciones integradas en ellos se haya hecho de una manera longitudinal y que, por tanto, el comparar los registros kénticos de los ss. XII y XIV (etapas temprana y tardía del IM) constituyera el segundo de los objetivos del presente trabajo.

Mediante el análisis de las variantes formales de los topónimos kénticos de los siglos XII y XIV, se intenta no sólo descubrir posibles patrones distribucionales de estos sonidos, sino también complementar y comparar esta evidencia con la información proporcionada tanto por estudios de corte diacrónico o tradicional, como de aquellos en los que se aprecia un enfoque sincrónico.

A la hora de recopilar el material de una investigación dialectológica basada en elementos toponímicos, debemos tener en cuenta que este debiera ser recogido de aquellos documentos locales que reflejen los desarrollos dialectales de la zona en cuestión con un grado de fiabilidad óptimo. Parece haber consenso en que los documentos que mejor cumplen dichas condiciones son los Subsidy Rolls, cuyo registro, aunque data de la época de Enrique II (1133-1189) no se regularizó hasta finales del siglo XIII (concretamente el año 1290). Este hecho junto a otras ausencias significativas ya mencionadas con anterioridad, hizo que se optara aquí por lo que, hasta el momento, constituye el registro más valioso para el propósito de cualquier investigación sobre la toponimia de Kent, esto es, la extensa obra de Wallenberg, Places-Names of Kent ${ }^{6}$.

\section{ANÁLISIS TOPONÍMICO}

Esta investigación se centró concretamente en el estudio de ocho variables del IA: $a$, $c e, e o, y$ (larga y breve), y las fricativas iniciales /f-, s-, $\theta-/$, que se han venido considerando

\footnotetext{
${ }^{6}$ Conjuntamente al uso de esta fuente primaria se consultaron también Anderson (1934-1939), Cameron (1961), Reany (1964), Copley (1968) y Ekwall (1980).
}

Odisea, $n^{\circ}$ 2, 2002 
dialectalmente relevantes en el caso de Kent. Como se ha apuntado anteriormente, y de acuerdo con la doctrina tradicional ${ }^{7}$ para los sonidos vocálicos simples arriba mencionados, es evidente que el desarrollo $e$ ha sido generalmente dominante en Kent, aunque esté ausente, o en gran minoría, con respecto a los desarrollos encontrados en el resto del país. En cuanto a los diptongos se refiere, éstos aparecen en Kent con representaciones digráficas prototípicas que podrían cuando menos indicar, aunque es materia de debate, un retraso en la lógica y normal monoptongación experimentada por la mayoría de los dialectos. En lo que respecta a las fricativas iniciales, tradicionalmente se ha considerado a Kent como una zona caracterizada por la sonoridad de éstas.

Una vez recopilados aquellos topónimos kénticos que en su etimología se componen de las variables del IA antes mencionadas, se procedió a su análisis, distinguiendo previamente entre los datos relativos al s. XII y los del s. XIV (dentro de cada siglo se hizo a su vez, una distinción entre lo que hemos dado en llamar un primer y un segundo elemento, según la variante bajo análisis perteneciera a un primer o segundo constituyente ${ }^{8}$ de un topónimo compuesto). Con los resultados obtenidos se hizo una valoración, siempre que ello resultó factible, de la coincidencia o discrepancia entre éstos y los aportados por autores como Jordan-Crook (1925 / 1974), Moore, Meech y Whitehall (1935), Ek (1972 y 1975), Kristensson (2001), amén de contrastar con los datos aportados por el LALME.

\section{ESTUDIO COMPARATIVO}

Por lo que a mis datos concierne, hay una coincidencia con Ek (1975), el LALME (1986) y Kristensson (2001) en cuanto al desarrollo en IM de la $c$ y la $y$ (larga y breve) del IA se refieren, ya que, en líneas generales, el resultado obtenido fue el de la típica $e$ kéntica mayoritaria. Son sin embargo dignos de destacar:

i. La presencia para la $a$ de algunos desarrollos en $a$ que pueden no deberse a un acortamiento $^{9}$ (véanse los ejemplos de la tabla $1^{10}$ ), algunos desarrollos en $i^{11}$ (véase tabla 2) y otros en $o$ (véanse a modo de ilustración los topónimos de la tabla 3). Pese a su probable interés, estos desarrollos no nos permiten elaborar hipótesis rigurosas, dadas su naturaleza circunstancial y su dispersa distribución geográfica.
IA hlcefdige 'dama'
LADYDOWN FM.
Ladydoune 1356 AD, v. 3
IA gemoere 'frontera'
MARLEY COURT
Marleye $1316 \mathrm{Cl}$

Tabla 1. Topónimos Kénticos con la variante $a$

\footnotetext{
$\overline{7}$ Luick (1914/1941), Wyld (1927), Oakden (1930), Moore, Meech y Whitehall (1935), Mossé (1952), Lass (1969), y Jordan-Crook (1925/1974).

8 Por constituyente nos referimos al elemento o término léxico que incorpora el sonido del IA que se analiza en cada caso.

${ }^{9}$ Véase con respecto a los desarrollos con la variante $a$, Wyld (1927: §62 (2)).

${ }^{10}$ Las tablas que a continuación presento se organizan en tres columnas:

i. La primera se compone del término léxico del IA que contiene la variable en cuestión, junto con su significado en español.

ii. La segunda consta de aquellas localidades kénticas (con su nombre moderno) que etimológicamente derivan de dicho término léxico.

iii. En la tercera se listan las diferentes formas toponímicas que dicha localidad tenía en la época bajomedieval. Aparte de resaltar en negrita la variante gráfica pertinente en cada caso, cada forma ha sido debidamente registrada con la fecha y la fuente correspondientes.
}

Odisea, $\mathrm{n}^{\circ} 2,2002$ 

IA loes 'pasto'
LEESDEN
Liseden 1338 Subs
LESSNESS HEATH
Lisna c. 1100 Text Roff
IA hwate 'trigo'
LITTLES FM.
Lidle 1327 Subs; Lydles 1373 Subs
WHITEACRE
Whitacre 1346 FA

Tabla 2. Topónimos Kénticos con la variante $i$
IA hap 'brezal'
LEWIS HEATH
Leneshothe 1317 FF; de Leues-hothe 1327
Subs; de Leneshothe 1331 Pat;
Leueshoth', -hothe 1332 Subs; Leueshothe
IA doel 'parte, 1338, 1347 Subs; Leuweshothe 1348 Subs
TWYDALL Twidole 1327 Subs división'

Tabla 3. Topónimos Kénticos con la variante $o$

ii. El predominio del desarrollo $i$ para la $y$ (larga y breve) del IA en la zona oriental de Kent durante el s. XII, y que, paradójicamente, coincide con el LALME (véanse los ejemplos de la tabla 4).

\begin{tabular}{|c|c|c|}
\hline IA brycg 'puente' & ELBRIDGE & Thelebrigge $1187 \mathrm{P}$ \\
\hline \multirow[t]{3}{*}{ IA cyning 'rey' } & CHINGESFORD & Chingesford 1166-7 P; King-hamford \\
\hline & & $1178-9 P$ \\
\hline & KINGSTON & Kyngestun $11 \mathrm{DM}$ \\
\hline \multirow[t]{5}{*}{ IA cyrice 'iglesia' } & CHERITON & Ciriceton c. 1100 Dom Mon; Ciretona \\
\hline & & 1174-5 P; de Ciriton' 1175-6 P. \\
\hline & EASTCHURCH & Eastcyrce c. 1100 Dom Mon \\
\hline & IVYCHURCH & Inecirce $11 \mathrm{DM}$ \\
\hline & WOODCHURCH & Wudecirce c. 1100 Dom Mo \\
\hline IA hyll 'colina' & HERNHILL & Haranhylle c. 1100 Dom Mon \\
\hline IA hyrne 'esquina' & HERNE & ate Hyrnan c. 1100 Dom Mon \\
\hline IA mynster & MINSTER & Sexburgamynster c. 1100 Dom Mon \\
\hline
\end{tabular}

Tabla 4. Topónimos con la variante $i$ 
iii. La frecuencia para la $y$ (larga y breve) de desarrollos con $u$ en el s. XIV, y cuya motivación puede residir más en el factor cronológico que en su composición léxica $^{12}$. Véase al respecto la tabla 5.

IA byrh 'burgo' GT. BURSTED de Burstede 1327, 1332, 1334, 1338 Subs; Burstede

FM. $\quad 1313$ Ass

BINBURY MA. Bengebury 1319 FF; 1335 Ipm; 1367 BM I; 1380

Cl; Byngebury $1319 \mathrm{Cl}$

HULBERRY Holtysbury $1348 \mathrm{FF}$; Hultesbury $1361 \mathrm{Cl}$;

Holtesbury 1361 Ipm; Heltesbury 1371 Ipm

IA hyll 'colina' GOLDHILL HO. Goldhull' 1319 FF; Goldhull $1339 \mathrm{Cl}, 1338$ Pat

LOWFIELD ST. Lafhullestret 1308 AD, v.3. 1332 Subs

OATEN HILL de Othull 1327 SR

IA hyrst 'bosque' BUCKHURST de Bokhurst 1338 Subs; ate Bokhurst 1347 Subs

YARDHURST de Yardhurst 1327 Subs

Tabla 5. Topónimos con la variante $u$

Por otro lado, nuestras formas toponímicas parecen diferir de los análisis tradicionales en:

i. La $c$ del IA, por la abundancia de formas con $a$ que ya desde el s. XII, especialmente en el primer o único elemento de los topónimos de la zona norte, parecen estar bien implantadas (el LALME y Kristensson (2001) coinciden con nuestras formas toponímica del s. XIV en tener también este desarrollo como mayoritario ${ }^{13}$. A modo ilustrativo, véase la tabla 6 . Pese a la constante presencia de $a$, es cierto que las formas con $e$, hasta después del $\mathrm{s}$. XIV, muestran cierta resistencia al lento, pero a la vez constante, proceso de decaimiento que ya se constata en el s. XII. Es por esto por lo que en el segundo constituyente de los topónimos compuestos, la probable pérdida de su acentuación y el consiguiente debilitamiento de las sílabas finales tuvo forzosamente que favorecer la resistencia de dicha $e$ (véase, a modo de ejemplo, la tabla 7).

\footnotetext{
11 Véase con respecto a los desarrollos con la variante $i$, Ek (1975: 35).

12 Aunque para Ek (1972) los desarrollos con $u$ aparecen sobre todo en topónimos compuestos por determinados términos léxicos (i.e., IA byrh, hyll y hyrst), la verdad es que entre nuestras formas dicha variable parece depender del factor cronológico pues, con la única excepción del IA hyll, los demás componentes muestran en el s. XII una tendencia hacia la variante $e$.

13 Véase Martín Díaz (2001).
} 

IA cecer 'acre'
TENACRE
de Tyenacr' 1327, 1332 Subs; de Tyenakre 1338 Subs; Tenakere 1347 Subs
IA cesc 'fresno' ASH
Sardasse 1197 FF
NASH'S FM.
Asshele $1363 \mathrm{Cl}$
IA *becstan
BAXON
Baxton', 1334 Subs; Baxtone, Bac-
'disco'
ston' 1338 Subs; Baxtone, -ton'
1347 Subs; Baxton’ 1348 Subs

IA blace 'negro' BLAXLAND FM. Blakyslonde $1308 \mathrm{AD}$, v. 6; atte Blakelonde, de Blakyslond, Joh. Blake 1332 Subs; de Blakeslond', Th., Gold; Blak' 1334 Subs; de Blakislonde 1346 Subs; de Blakeslonde 1347 Subs

IA $d c e l$ 'valle' WORMDALE Wormedale $1195 \mathrm{Abbr}$; Wermedale 1194 P; de Wermedal' 1198 P; Wrmedale, Wormedale, Wyrmedale 1313 Ass; Wormedale 1327 Subs; de Wormendale 1329 Ipm DALHAM de Dalham 1313-4 Seld 24, 200; 1313 Subs

IA fager 'bello' FAIRBROOK de Fayr-, Fairbrok 1318 Pat; ate FM. ffayrebrok' 1332 Subs

IA gewaed IWADE Atwangeraede 11 DM, Ywada 'vado' $1179 P$

IA haecc 'puerta' HATCHMAN Hac(c)hesham 1334 FF; 1369 , 
'cuervo'

IA moegden

'doncella'

'promontorio'

IA smael

'pequeño'

IA woeter 'agua' WATCHESTER

FM.

WATERHAM

WATERHAM FM MAYTON FM.

MARSHES

Rammesgate 1304 Ass; de Ramesgat' 1334 Subs; de Ramesgate 1357 Subs de Maiham c. 1185 Pensh; Mayton 1308 AD, v. 6; de Maytone 1332 Subs; Mayton 1343, 1344 Cl; Maytham(e) 1357, 1393 Pat

TAILNESS Taylnesse 1368 Pat

SMALLDANE Joh. Smaldane 1348 Subs

IA Apelnop / ALLINGTON Alnodentune c. 1100 Dom Mon;

Agelnop,Pers. N Alnothintone 1308 Ipm; Ailnothintone 1327 Subs

IA $\operatorname{Wed}(d) a, \quad$ WEDDINGTON de Wadyntone 1348 Subs Pers. N 
IA cecer 'acre' GREENACRE FM. de Grimesekere 1304 Ass

\begin{tabular}{|c|c|c|}
\hline & ROMACRES & de, atte Romekere 1327 Subs \\
\hline IA cern 'casa' & TYLERHILL & $\begin{array}{l}\text { Tylerhelde } 1304 \text { Ass, Teghelere-helde 1363, } \\
\text { 1379 Subs }\end{array}$ \\
\hline IA doel 'valle' & TUDELEY & $\begin{array}{l}\text { Tiuedele c. } 1100 \text { Dom Mo; Thewdele } 1313 \text { Ass, } \\
\text { Teudele 1316, } 1320 \mathrm{FF} \text {; 1324-5 BM I; } 1324 \mathrm{Cl} \text {; } \\
1327 \text { Ipm; Teudele, Tyedele } 1353 \mathrm{FF}\end{array}$ \\
\hline $\begin{array}{l}\text { IA gehoeg } \\
\text { "cercado" }\end{array}$ & ROUGHWAY & Rogheye 1313 Ass \\
\hline IA haecc 'puerta' & TRAMHATCH & de Thremhecch' 1338 Subs \\
\hline $\begin{array}{l}\text { IA ncess } \\
\text { 'promontorio' }\end{array}$ & SHEERNESS & Sirnesse 1329 Ch \\
\hline $\begin{array}{l}\text { IA *Eadmoer, } \\
\text { Pers. N. }\end{array}$ & ADMER'S WOOD & Edmeris field $1381 \mathrm{Cl}$ \\
\hline
\end{tabular}

Tabla 7. Topónimos con la variante $e$

ii. Los diptongos ea y eo del IA, porque en ambos es precisamente $e$ el desarrollo predominante. En el caso del diptongo ea la e quizá se explique debido al abundante número de topónimos compuestos por la combinación $e a+h$ en la que e es el desarrollo esperado en Kent (véase tabla 8). Con respecto a IA eo, véanse los ejemplos de la tabla 9.

\begin{tabular}{|c|c|c|}
\hline IA ceap 'barato' & CHIPSTEAD & $\begin{array}{l}\text { Chepsteda } 1191 \text { P; de Chepstede } \\
1313 \text { Ass }\end{array}$ \\
\hline IA east 'este' & EASTWELL & Estwelle c. 1100 Dom Mon \\
\hline \multirow[t]{3}{*}{ IA heah 'alto' } & $\begin{array}{l}\text { HIGHAM } \\
\text { (HADLOW) }\end{array}$ & $\begin{array}{l}\text { de Hegham 1327, 1332, } 1334, \\
\text { 1338 Subs; de Heghham } 1332 \\
\text { Subs }\end{array}$ \\
\hline & HIGH REED & Heghrede $1381 \mathrm{Cl}$ \\
\hline & HIGHSTEAD & Heghsted 1314 Pat \\
\hline \multirow[t]{4}{*}{ IA leah 'prado' } & BROCKLEY & $\begin{array}{l}\text { Brocele 1182-4 Reg Roff; } \\
\text { Brockele 1328 Ch }\end{array}$ \\
\hline & HAWLEY & $\begin{array}{l}\text { Hageleg c. } 1100 \text { Dom Mon; } \\
\text { Halgelei c. } 1100 \text { Text Roff; } \\
\text { Halgele 1199 Cur }\end{array}$ \\
\hline & LEIGH GREEN & Lega c. 1100 Text Roff \\
\hline & SWANLEY & Swanle 1396 Pat \\
\hline $\begin{array}{l}\text { IA Eadmoer, } \\
\text { Pers. N. }\end{array}$ & ADMERS WD. & $\boldsymbol{E}$ dmeris field $1181 \mathrm{Cl}$ \\
\hline
\end{tabular}

Tabla 8. Topónimos con la variante $e$ 


\begin{tabular}{|c|c|c|}
\hline IA beonet 'juncos' & BENSTED & $\begin{array}{l}\text { Bedenestede c. } 1100 \text { Dom Mon; } \\
\text { de Bentested' } 1199 \text { FineR; } \\
\text { Bentestede } 1312 \text { FF; Bentsted } \\
\text { 1316 Ipm }\end{array}$ \\
\hline IA breosa 'tábano' & BRISHING WD. & $\begin{array}{l}\text { Bresyng } 1346 \mathrm{FA} ; \quad \text { Bresynge } \\
1362 \mathrm{Cl} \text {; Bressing' } 1368 \mathrm{Cl} \text {; } \\
\text { Bressinge Ipm }\end{array}$ \\
\hline $\begin{array}{l}\text { IA deop } \\
\text { 'profundo' }\end{array}$ & DIBDEN & $\begin{array}{l}\text { de Depedene } 1310 \text { FF; } 1332 \\
\text { Subs; de Depeden' } 1315 \text { FF; de } \\
\text { Depedene } 1327 \text { Subs; Depe- } \\
\text { denn' } 1334 \text { Subs; de Depedenne } \\
\text { 1338 Subs; Depinden', Depe(n)- } \\
\text { den' (p.) 1348 Subs }\end{array}$ \\
\hline $\begin{array}{l}\text { IA deor 'ciervo, } \\
\text { animal' }\end{array}$ & DARGETS WD. & de Dergate 1313 Ass \\
\hline IA neowe 'nuevo' & NEWCOURT $W D$. & $\begin{array}{l}\text { Newecourt } 1327 \text { Subs; 1343-4 } \\
\text { Ass; 1343-4 Ass; de Neweco(u)rt } \\
\text { 1332, 1334, 1338 Subs; Newe- } \\
\text { court 1346 FA }\end{array}$ \\
\hline & NEWLANDS & $\begin{array}{l}\text { Atte Newelonde 1327. 1332, } \\
1334,1347 \text { Subs }\end{array}$ \\
\hline $\begin{array}{l}\text { IA preosta } \\
\text { 'párroco' }\end{array}$ & $\begin{array}{l}\text { NEWNHAM FM. } \\
\text { PRESTON FM. }\end{array}$ & $\begin{array}{l}\text { De Newenham } 1332 \text { Subs } \\
\text { Preston' } 1377 \text { FF; Preston } 1325 \\
\text { Cl; } 1330 \text { Ipm; de Prestone } 1332 \\
\text { Subs; de Preston' } 1334 \text { Subs; } \\
\text { Preston } 1346 \text { FA; } 1372 \text { Cl }\end{array}$ \\
\hline
\end{tabular}

Tabla 9. Topónimos con la variante $e$

iii. Las fricativas iniciales, porque contrariamente a lo que cabría esperar, la presencia de una sonorización en las mismas no parece ser precisamente el rasgo más distintivo en el Kent de la época, al menos en lo que a los topónimos se refiere. El carácter unitario del que hablan algunos autores, por tanto, queda descartado en este análisis, ya que dicho proceso parece afectar, única y superficialmente, a la /f-/ durante el s. XIV.

\section{CONCLUSIÓN}

Los fonemas del IA aquí tratados presentan en Kent un desarrollo durante el IM que en ocasiones coincide con el constatado previamente para este condado, pero que en otras muchas, difiere de la variedad formal que, a veces de manera superficial, se ha interpretado como típicamente kéntica. Aunque se ha hablado ya de los inconvenientes que una investigación como ésta, basada en un estudio toponímico, pudiera tener, hemos podido 
observar también cómo el análisis de algunos escasos textos ancla tampoco es suficiente para hacer ciertas generalizaciones dialectales. El que la realidad reflejada en los topónimos que aquí se presentan sea en ocasiones más similar a la encontrada en el $L A L M E$ (que se ha encargado de paliar este problema usando un número más elevado de textos "localizables") que a la tradicionalmente expresada, es indicativo de que, con el fin de llegar a un mejor entendimiento de etapas pasadas de la lengua y sobre todo de las variedades dialectales que en ella se daban, las fuentes documentales o literarias deben ser, siempre que se pueda, contrastadas con el material onomástico correspondiente.

\section{BIBLIOGRAFÍA}

Arngart, O. S. (Anderson). 1949. "Middle English dialects". Studier i Modern Sprakvetenskap XVII: 17- 29.

Benskin, M. 1991 (1989). “The 'fit'-technique explained”. Ed. F. Riddy. Cambridge: Brewer. 9-26.

Benskin, M. y M. Samuels, eds. 1981. So Meny People Longages and Tonges: Philological Essays in Scots and Mediaeval English Presented to Angus McIntosh. Aberdeen: Aberdeen University Press.

Blake, N. F. 1981. Non-Standard Language in English Literature. Londres: Andre Deutsch.

ed. 1992. The Cambridge History of the English Language: 1066-1476. Volumen II. Cambridge: Cambridge University Press.

Brook, G. L. 1965 (1958). English Dialects. Londres: Andre Deutsch.

Cameron, K. 1961. English Place-Names. Londres: Batsford.

Copley, G. J. 1968. English Place-Names and their Origins. Devon: David \& Charles.

Ek, K.G. 1972. The Development of $O E y$ and eo in South-Eastern Middle English. Lund: Gleerup.

1975. The Development of $O E$ ce (i-mutated a) before Nasal and $O E$ in South-Eastern Middle English. Lund: Gleerup.

Ekwall, E. 1931. Studies on English Place- and Personal Names. Lund: Gleerup. Oxford: O.U.P.

1980 (1940, 1960). The Concise Oxford Dictionary of English Place-Names.

Gradon, P.O.E., ed. 1979. Dan Mitchel's Ayenbite of Inwyt. Early English Text Society 278.

Heuser, W. 1895. "Zum Kent dialekt im Mittelenglischen.” Anglia Anzeiger XVII: 7390.

Hogg, R. M. 1992. A Grammar of Old English. Vol. I. Oxford: Blackwell. . ed. 1992. The Cambridge History of the English Language: 1066-1476. Vol. I. Cambridge: C.U.P.

Jordan, R. 1974 (1925, 1934). Handbook of Middle English Grammar: Phonology. (Trad. y rev. por E. J. Crook). La Haya: Mouton. [Jordan-Crook]. 
Kristensson, G. 1967. A Survey of Middle English Dialects 1290-1350: The Six Northern Counties and Lincolnshire. Lund: Gleerup.

. 1976. "Lay subsidy rolls and dialect geography". English Studies 57: 51-59. 1987. A Survey of Middle English Dialects 1290-1350: The West Midland Counties. Lund: Gleerup.

1995. A Survey of Middle English Dialects 1290-1350: The East Midland Counties. Lund: Wallin \& Dalholm Boktr AB.

2001. A Survey of Middle English Dialects 1290-1350: The Southern Counties: Vowels (except Diphthongs). Lund: Lund University Press.

Lass, R., ed. 1969. Approaches to English Historical Linguistics: An Anthology. Nueva York: Holt, Rinehart and Winston.

Luick, K. 1941 (1914). Historische Grammatik der Englischen Sprache. Stuttgart: Tauchnitz.

Martín Díaz, M. A. 2001. "A two-fold development for OE æ in $12^{\text {th }}$ - and $14^{\text {th }}$-century Kentish place-names". English Place-Name Society 33: 21-54.

McIntosh, A. 1956. "The analysis of written Middle English". Transactions of the Philological Society 55: 26-55. Reimpresión Lass (1969: 35-57). $1-11$.

. 1963. "A new approach to Middle English dialectology". English Studies 44:

McIntosh, A., M. L. Samuels, M. Benskin, M. Laing y K. Williamson. 1986. A Linguistic Atlas of Late Mediaeval English. Aberdeen: Aberdeen U.P. [Abrev. LALME].

McIntosh, A., M. L. Samuels y M. Benskin. 1987. Guide to a Linguistic Atlas of Late Medieval English. Aberdeen: Aberdeen U.P.

Milroy, J. 1992. Linguistic Variation \& Change. Oxford: Basil Blackwell. 1992. "Middle English dialectology". The Cambridge History of the English Language: 1066-1476. Vol. II. Ed. N. F. Blake. Cambridge: Cambridge University Press. 156-206.

Moore, S., S. B. Meech y H. Whitehall. 1935. "Middle English dialect characteristics and dialect boundaries". Essays and Studies in English and Comparative Literature. Ann Arbor: University of Michigan Publications. 1-60.

Morris, R. y P. Gradon, eds. 1866. Dan Michel's Ayenbite of Inwyt. Early English Text Society (Original Series) 23.

Morsbach, L. 1888. Über den Ursprung der Neuenglischen Schriftsprache. Heilbronn.

Mossé, F. 1952. A Handbook of Middle English. Londres: The Johns Hopkins University Press.

Oakden, J. P. 1930. Alliterative Poetry in Middle English. 2 vols. (Vol. I, Part I: "The dialectal survey". 5-39). Manchester: Manchester University Press.

Reany, P. H. 1964 (1960). The Origin of English Place-Names. Londres: Routledge \& Kegan Paul. 
Riddy, F. ed. 1991 (1989). Regionalism in Late Medieval Manuscripts and Texts: Essays Celebrating the Publication of A Linguistic Atlas of Late Mediaeval English. Cambridge: Brewer.

Rubin, S. 1951. The Phonology of the Middle English Dialect of Sussex. Lund: Carl Bloms Boktryckeri AB.

Samuels, M. L. 1969. “Some applications of Middle English dialectology”. Ed. R. Lass. Nueva York: Holt Rinehart and Winston. 404-18. . 1972. Linguistic Evolution. Cambridge: C.U.P.

Serjeantson, M. S. 1922. "The dialectal distribution of certain phonological features in Middle English". English Studies 4: 93-109, 191-98. . 1924. Distributions of Dialect Characters in Middle English. Amsterdam. . 1927a. "The dialects of the West Midlands in Middle English". Review of English Studies 3: 54-67, 186-203, 319-31. . 1927b. "The development of Old English eag, eah in Middle English". Journal of English and Germanic Philology XXVI: 198-225, 350-400.

Sundby, B. 1963. Studies in the Middle English Material of Worcestershire Records. Bergen - Oslo: Norwegian University Press.

Taylor, A. B. 1924. "On the history of OE ea, eo in Middle Kentish”. Modern Language Review 19: 1-10.

Wallenberg, J. K. 1923. The Vocabulary of Dan Mitchel's Ayenbite of Inwyt. Uppsala: Appelbergs Boktryckerei Aktiebolag. Aktiebolag.

. 1934. The Place-Names of Kent. Uppsala: Appelbergs Boktryckerei

Wyld, H. C. 1913. "The treatment of OE y in the dialects of the Midland and South Eastern counties in ME; and of OE y in the dialects of the South and South Western counties in ME". Englische Studien 47: 1-58.

. 1921. "South-Eastern and South-East Midland dialects in Middle English". Essays and Studies VI: 112-45.

. 1927 (1914). A Short History of English. Londres: John Murray. 